\title{
Food Adulteration: From Vulnerability Assessment to New Analytical Solutions
}

\author{
Christophe Cavin*, Geoffrey Cottenet, Carine Blancpain, Thomas Bessaire, Nancy Frank, \\ and Pascal Zbinden
}

\begin{abstract}
Crises related to the presence of melamine in milk or horse meat in beef have been a wake-up call to the whole food industry showing that adulteration of food raw materials is a complex issue. By analysing the situation, it became clear that the risk-based approach applied to ensure the safety related to chemical contaminants in food is not adequate for food fraud. Therefore, a specific approach has been developed to evaluate adulteration vulnerabilities within the food chain. Vulnerabilities will require the development of new analytical solutions. Fingerprinting methodologies can be very powerful in determining the status of a raw material without knowing the identity of each constituent. Milk adulterated by addition of adulterants with very different chemical properties could be detected rapidly by Fourier-transformed mid-infrared spectroscopy (FT-mid-IR) fingerprinting technology. In parallel, a fast and simple multi-analytes liquid-chromatography tandem massspectrometry (LC/MS-MS) method has been developed to detect either high levels of nitrogen-rich compounds resulting from adulteration or low levels due to accidental contamination either in milk or in other sensitive food matrices. To verify meat species authenticity, DNA-based methods are preferred for both raw ingredients and processed food. DNA macro-array, and more specifically the Meat LCD Array have showed efficient and reliable meat identification, allowing the simultaneous detection of 32 meat species. While the Meat LCD Array is still a targeted approach, DNA sequencing is a significant step towards an untargeted one.
\end{abstract}

Keywords: Adulteration · Fraud · Meat · Milk · Nitrogenous compounds

\section{Introduction}

Intentional adulteration of food for financial advantage has been performed for centuries worldwide..$^{[1,2]}$ Although the motivation is purely economic, it is important to remember that adulteration is a criminal act which may result in significant public health concern. ${ }^{[3]}$ Melamine's crisis is still in everybody's mind, however, new cases are regularly identified. Striking examples of new possible harm related to food fraud are the use of protein hydrolysates extracted from residues of the leather industry which may contain highly toxic chromium(VI). An other example is the use of recycled oil or gutter oils. ${ }^{[4,5]}$ Management of food safety risks often focuses on hazards that occur naturally in foods or that are unintentionally added by food processing, storage or handling within the food production chain. Therefore, our expertise and science is normally used to predict issues and focus our food quality and safe-

${ }^{\star}$ Correspondence: Dr. C. Cavin

Nestle Research Center

P.O. Box 44, Vers-chez-les Blanc

$\mathrm{CH}-1000$ Lausanne 26

E-mail: christophe.cavin@rdls.nestle.com ty activities. In food adulteration, we are facing the unknown and the wide range of possibilities or fraudulent acts that can be used on raw materials makes traditional risk-based preventive control systems inapplicable. ${ }^{[6-8]}$ In this context, it is important to know what drives the fraudsters, to better understand the food vulnerabilities to adulteration since suitable targets provide opportunities for fraud. In the case of food adulteration, suitable targets can be ingredients, raw materials and products which are by the nature of their composition, their processing level, their qualities or geographical origin particularly attractive for fraud. Opportunities are associated with specific raw materials vulnerabilities that are exploited by fraudsters. ${ }^{[9]}$ The possibilities for adulteration encompass certain categories including the deliberate substitution, dilution, unapproved enhancement, concealment or mislabelling of food ingredients. ${ }^{[2,10]}$

\section{Understanding the Raw Material Vulnerabilities}

\section{Vulnerabilities Driven by Factors Inherent to the Raw Material}

A general approach to prevent food adulteration can be summarised as know your raw materials and its vulnerabilities, your supply chain, suppliers and verification measures. In this context, it is impor- tant to understand first the raw material's inherent vulnerabilities. They are dependent mainly on the raw material physical state (e.g. liquid, solid, powder), its level of processing and its composition (e.g. fat, protein, carbohydrates content). These vulnerabilities are driven by factors totally inherent to the raw material and thus, are entirely independent of the actions taken by the buyers to mitigate the risk of food fraud. Some raw materials are by nature more vulnerable to adulteration, e.g. fruit juices and purees are more vulnerable than fruit pieces. ${ }^{[7]}$

The level of processing is a key parameter influencing the raw material vulnerabilities. As shown in Fig. 1, vulnerabilities are significantly increased with processing. It goes from mislabelling of wild by farmed fish and possible species substitution (if the whole fish is purchased by a non-expert) to several potential fraudulent practices when the fishes are processed to fillets (single or block) or to fish powders, e.g. mislabelling, species substitution, dilution using water-retention agents, concealment by addition of illegal preservatives or flavours, unapproved enhancement of protein level in powders.

In the context of an economically motivated adulteration, the raw material price is clearly an important parameter of raw material inherent vulnerability. However, more than just the raw material market price, it is also the volume purchased and 


\begin{tabular}{|c|c|c|c|}
\hline \multirow{7}{*}{$\begin{array}{l}\text { Whole fish } \\
\text { Mislabeling } \\
\text { - Farm/wild } \\
\text { Substitution } \\
\text { - Species }\end{array}$} & & \multirow{7}{*}{$\begin{array}{l}\text { Block of fillets } \\
\text { Mislabeling } \\
\text { Substitution } \\
\text { Concealment } \\
\text { Dilution } \\
\text { - Water retention }\end{array}$} & \multirow{7}{*}{$\begin{array}{l}\text { Fish powder } \\
\text { Mislabeling } \\
\text { Substitution } \\
\text { Dilution } \\
\text { - Fillers } \\
\text { Concealment } \\
\text { - Flavors } \\
\text { Unapproved enhancemen } \\
\text { - Proteins }\end{array}$} \\
\hline & & & \\
\hline & Single fillet & & \\
\hline & Mislabeling & & \\
\hline & Substitution & & \\
\hline & Concealment & & \\
\hline & - Dyes, preservatives & & \\
\hline & reased & vulnera & \\
\hline
\end{tabular}

Fig. 1. Analysis of the inherent fish vulnerabilities to adulteration. A significant increase is observed with fish processing from whole fish to fish powder.

payment criteria of this raw material which strongly influence its inherent vulnerabilities. A good example is raw milk for which the market price is not high. However, it is a raw material which has been highly adulterated worldwide for many years. Its physical state as a liquid makes it easy to adulterate by addition of many adulterants such as water, urine or using milk from foreign species. Raw milk is not only paid to farmers based on the volume provided but also on its composition (e.g. protein and fat levels) and on its microbiological quality. This has a strong impact on the milk's inherent vulnerability to adulteration since many compounds with very different physico-chemical properties can be added to make profit on milk. ${ }^{[11]}$

Looking at the raw material fraud history or past cases of adulteration is a good source of information. ${ }^{[9]}$ It will help to identify illegitimate practices applied previously to specific raw materials or food products. Moreover, it is a good source of information showing how fraudsters may play on specific payment criteria of the raw material such as an unapproved increase of protein levels. The melamine crisis showed that non-protein nitrogen (NPN) can be used if the analysis of the raw material protein level is not based on the 'true' protein content, thus opening the door to a whole range of new adulterants rich in nitrogen compounds. New analytical developments regarding this concern will be discussed later.

\section{Vulnerabilities Driven by Factors Impacting the Business (Business Pressure)}

While all foods and food ingredients have the potential to be a target for fraud, public health concerns and economic impact may differ significantly. Therefore, the business pressure for a large company working with several commodities needs oil) due to poor harvest caused by a new parasite and due to bad climatic conditions in a main country of production. This is a good example of increased raw material vulnerability based on rapid fluctuations of market pricing.

\section{Vulnerabilities Driven by Factors under the Control of the Buyer}

Further vulnerability assessment will reflect the strength or the weakness of a company's mitigation strategy, e.g. full traceability, adequate purchasing specifications, availability of analytical methods, and robustness of surveillance programs. In contrast to the inherent and business vulnerabilities described previously which are not or hardly controllable by the buyer, a lot of internal actions can be taken to better control these specific factors and decrease identified gaps and global vulnerability level as shown in Fig. 2 .

A streamlined upstream value chain improves traceability and transparency and gives fewer opportunities to fraudsters to act on the supply chain. ${ }^{[2]}$ Simplification and full mapping of the supply chain will significantly decrease vulnerabilities. Additionally, supplier readiness to share information on the supply chain and processes will help to increase the confidence level. Specification adequacy is also an important preventive aspect against food fraud. Raw material purchasing specifications must include appropriate authenticity criteria to mitigate as much as possible the inherent raw material vulnerabilities identified previously. Specification criteria linked to food fraud prevention must be thoroughly defined, in line with the level of complexity and variability of the ingredient's composition in order to accept only a natural inherent variability of the raw

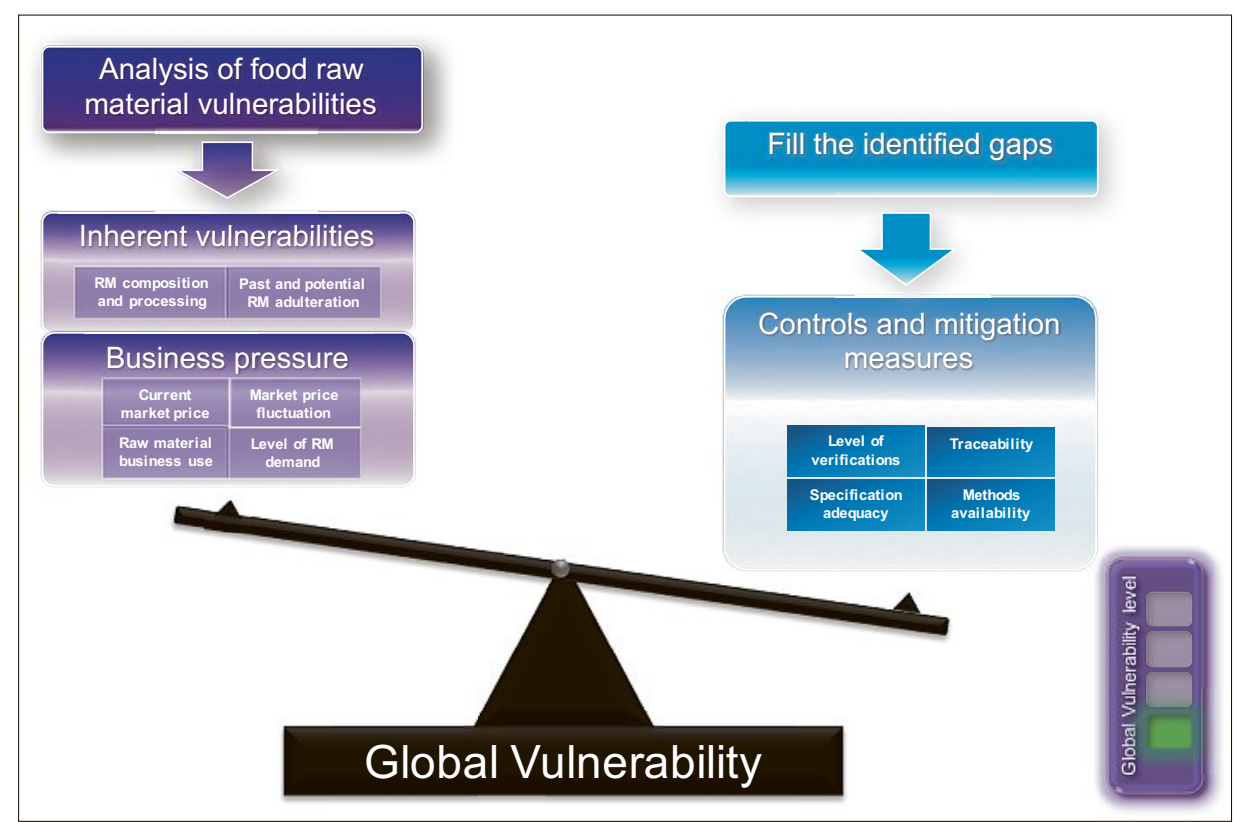

Fig. 2. Scheme describing the vulnerability assessment of food raw materials. Global food vulnerability reflects the balance between vulnerabilities which are not or hardly controllable versus the food fraud mitigation measures which are under the control of the buyer. 
material. When a specific parameter needs to be measured to control the raw material authenticity as part of the level of verifications performed by the buyer, attention must be given to use fit for purpose analytical methods with appropriate specificity and sensitivity for the control of authenticity.

\section{Significant Raw Material Vulnerabilities Leading to New Analytical Solutions}

\section{Meat and Poultry Vulnerabilities}

Meat and poultry are food categories that are highly vulnerable to adulteration. Looking at the main parameters influencing the vulnerabilities that have been mentioned previously such as the meat processing level, meat market price, meat demand and others, many opportunities for fraudulent activities can be identified. Meat species substitution was in the spotlight in 2013 due to the European horse meat crisis. ${ }^{[12]}$ However, several other types of fraud by substitution may also occur in meat and poultry food categories, e.g. by cheaper quality material from the same species, by less premium meat country of origin or meat label or by non-organic meat for organic one. When meat pieces are further processed, concealment using illegal preservatives, flavours or dyes as well as unapproved enhancement of ground meat, meat specialities or meat powder with nonmeat material or non-meat proteins (e.g. from vegetables) could be of next concern. The horse meat crisis had a critical impact on consumers' trust in this food sector, so species substitution is clearly a major concern for customers today. Many meat and poultry species commonly consumed by humans are quite expensive on the market and numerous less valuable meat species can easily be used as substitute. In this context severe cases have been encountered in South Africa and China (e.g. giraffe, fox). Therefore, new analytical methodology development was urgently required to face this issue.

\section{DNA-based Methods as the Reference Approach for Species Identification}

To verify meat species authenticity and traceability, many technologies have been described, such as two-dimensional electrophoresis, isoelectric focusing, protein capillary electrophoresis, HPLC, ELISA, and more recently peptide biomarker mass spectrometry. ${ }^{[13]}$ However, these approaches usually allow the identification of few meat species only. Also, to overcome limitations related to heat-treated or processed products, the use of DNA-based methods and especially Polymerase Chain Reaction (PCR) techniques are preferred for both raw ingredients and processed food. ${ }^{[14]}$

For meat species identification, the described PCR methods still present the same limitation: the majority of them target the expected meat species, like beef, pork, horse, chicken and turkey, and/or are limited to a maximum of 5-6 meat species. ${ }^{[15]}$ Taking into account the latest cases of meat adulteration in China where donkey and lamb meat were found to contain fox and rat meat, respectively, ${ }^{[16,17]}$ there is a strong need to expand the range of detectable meat species.

DNA macro-array, and more specifically a Meat Low Cost-low Density (LCD) Array, has already been evaluated by previous studies and showed efficient and reliable meat identification. ${ }^{[18]}$ This Meat LCD Array approach is based on classical PCR followed by a LCD array hybridisation. The PCR step amplifies a fragment ranging from 115 to 125 base pair (bp) from the vertebrates' $16 \mathrm{~S}$ rRNA mitochondrial gene, then amplicons are hybridised on a macro-array spotted with species-specific capture probes. This Meat LCD Array has recently been upgraded to allow the simultaneous detection of 32 meat species at a level of $1 \%$ w/w (Fig. 3)

While this Meat LCD Array is still a targeted approach, DNA sequencing can be considered as an untargeted one. Sanger DNA sequencing is now considered as the most reliable method for species identification on pure samples, especially for seafood identification. Known as DNA Barcoding, it is based on the International Barcode of Life initiative (iBOL), which developed an identification system for eukaryotic life based upon the analysis of sequence diversity in short, standardised gene regions (barcodes). A specific campaign, FISH-BOL, ${ }^{[19]}$ was initiated in
2005 and has barcoded more than 11000 fish species amongst the 32000 listed. For animals including fish, a fragment consisting of $\sim 700 \mathrm{bp}$ starting near the 5'-end of the cytochrome c oxidase subunit 1 (COI) mitochondrial gene has been selected. This region has been shown to reliably discriminate most commercial species of fish. ${ }^{[20]}$ DNA Barcoding is now frequently used to detect fish fraud, ${ }^{[21]}$ and recognised as the gold standard approach for fish species identification by the Food and Drug Administration (FDA). ${ }^{[22]}$ Although highly reliable to identify pure fish samples, experiments have shown that this technology is able to detect fish mixtures down to $5-10 \%$ w/w (Fig. 4), whereas the EU Recommendation 2013/99/EU requires a detection limit down to $1 \%$. Evolution of DNA sequencing to Next Generation Sequencing (NGS) should allow the detection and identification of lower amount of adulterants. Recent experiments of NGS on authenticity topics have shown promising results as an untargeted approach, ${ }^{[23]}$ which could be applied to spices, herbs or botanical supplements where risk of substitution and fraud are well-known especially in powder forms.

\section{Milk Vulnerabilities}

Milk vulnerabilities to adulteration are of major concern since milk is a key nutritional raw material widely used in infant and children products. Moreover, this group of population is particularly sensitive, being exposed via their significant daily milk consumption to higher concentrations of potential adulterants on a body weight basis. Although milk is not one of the most expensive raw materials, it has been adulterated for a very long time and is still cited as one of the top 10 raw materials most vulnerable to adulteration. Moreover, fraudulent cases are regular-

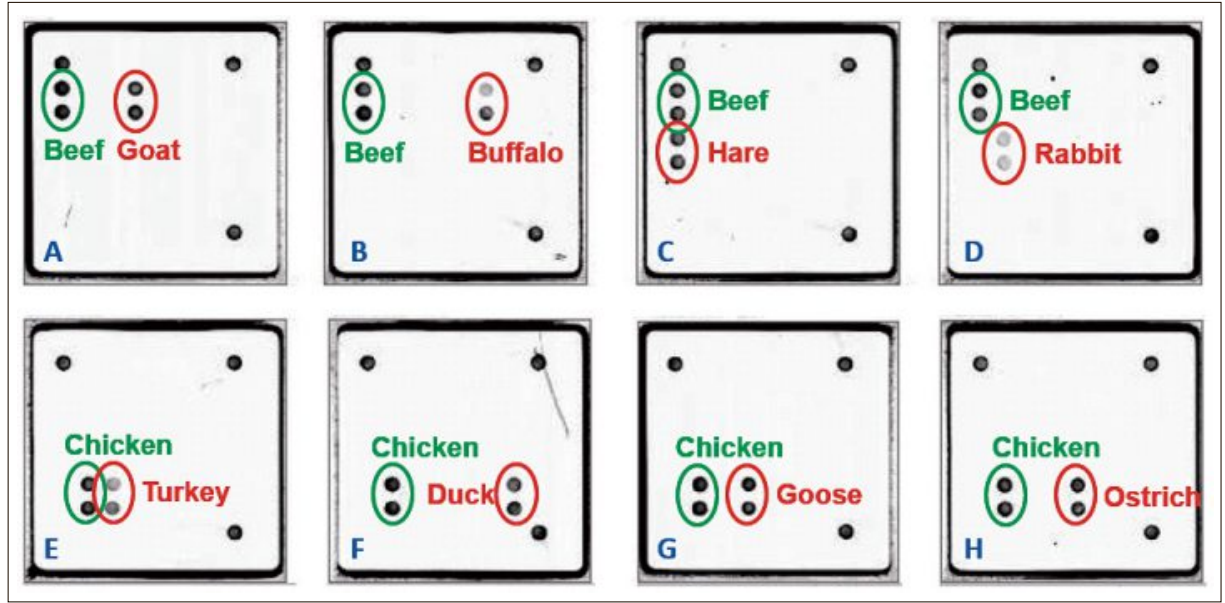

Fig. 3. Scan of a Meat LCD Array chip on which beef meat was mixed with $1 \%$ goat (A), water buffalo $(B)$, hare $(C)$, rabbit $(D)$, and chicken meat was mixed with $1 \%$ turkey $(E)$, duck $(F)$, goose $(G)$ and ostrich $(\mathrm{H})$. The dots present on three corners of each array correspond to hybridisation controls. Each species-specific probe is spotted in duplicate on each array (green and red circles). 


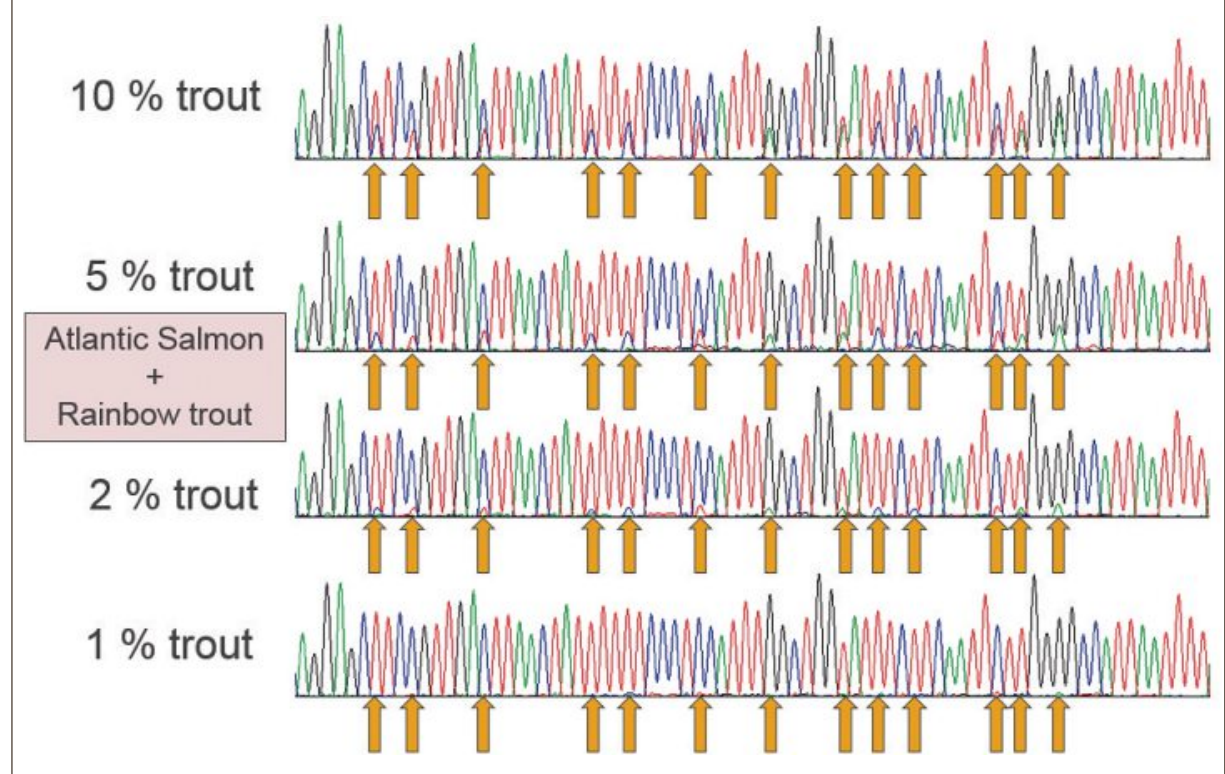

Fig. 4. Snapshot of chromatograms from Sanger DNA sequencing obtained on Atlantic salmon mixed with $1 \%, 2 \%, 5 \%$ and $10 \%$ of rainbow trout. Arrows indicate the position of different nucleotides between the two fish species.

ly reported worldwide.[24] Several factors may explain this. The milk supply chain is complex. This is particularly the case in some regions of the world where small farmers in large numbers are providing small quantities of milk at collection centres situated quite far from factories. This makes the control of the milk integrity very difficult. Moreover, the milk composition is naturally highly variable. This is due to several factors such as the seasonal feeding variations of the cows, milking from different cow species with cows at various stage of lactation and additionally, possible mixing in some countries of cow and buffalo milk which have different milk composition (e.g. fat and protein levels). This natural milk variability provides a lot of complexity to define the composition of an authentic milk in a specific region. Moreover, the criteria of payment of a raw material is a key parameter influencing its vulnerability to adulteration. In the case of milk, there are multiple criteria. Milk payment is not based only on the volume but the fat and protein levels and the bacteriological quality of milk are important factors. Therefore, all these parameters are susceptible to be exploited by fraudsters for economic gain. In this context, the possibilities to adulterate milk are high and easy to perform due to its liquid state. This has been confirmed by the significant number of milk adulterants identified from previous cases worldwide.

\section{New Analytical Approaches to Mitigate Risk of Milk Adulteration}

Currently mostly targeted methodologies have been developed in the food industry to verify milk integrity in addition to a measure of the proximates such as fat, protein, and non-fat solids essential for supplier payment. While this approach is very effective in screening out particular adulterants, the scenario is not applicable in a situation where the number of potential adulterants are significant and ever growing rapidly. It will become expensive and time consuming to have targeted methods focused on one adulterant at a time, therefore this approach is not applicable at milk collection centres or factories where farmers and lorries are waiting to deliver milk. This clearly highlights the need for new detection methodologies for countering milk adulteration. A more practical solution is to develop complementary fingerprinting or profiling technologies which can rapidly identify and differentiate the adulterated from the normal samples. These global fingerprints can be very powerful in determining the status of a raw material without knowing the identity of each constituent. For an application to milk, several criteria are required for technology selection such as a multi-residue approach, high-throughput, minimum sample preparation, low cost of operation and applicable in operational environment. Fourier transformed mid-infrared spectroscopy (FT-Mid-IR) is the technology which fits best all these criteria. ${ }^{[11,25]}$ Moreover, this instrument is often already present in milk factories for the determination of compositional parameters (e.g. fat, protein, non-fat solids) using specific regions of the spectrum. However, these instruments can also be used as fingerprinting tools together with chemometric data treatments to create a model of the normal milk profile. Samples are tested against this model allowing the detection of any significant changes in the spectrum and hence a potential adulteration. Spectral datasets using FT-Mid-IR (Milkoscan FT120) were gathered from different origins (Switzerland, India, and China). When Indian and Chinese milk spectra were projected into the Swiss model, significant differences could be observed between the fingerprinting models. For this reason, creating a global model pooling milk samples from different countries is not appropriate, there is a significant risk of decreasing the sensitivity of the methodology. The study performed on Swiss raw milk samples and artificially adulterated samples showed that milk adulterants with very different chemical properties can be detected rapidly by FT-Mid-IR fingerprinting (Fig. 5) showing the selection of milk adulterants representative for each class of concern. Data showed that this technology is more sensitive for the detection of small molecules and preservatives (0.003-0.15\%) whereas adulterants used to increase or correct the fat, protein and non-fat solid concentrations in milk are detected at higher levels $(0.15-1 \%$ or more depending on the adulterants). These results suggest that FT-Mid-IR fingerprinting technology is compatible with the detection of most milk adulterants at economic concentration. However, preservatives, neutralizers and antimicrobial agents can be effective in milk at much lower concentrations than detected with FT-Mid-IR thus requiring some targeted methods to complement the surveillance. Moreover, a semi-targeted rather than a completely non-targeted approach is currently under investigation using MALDI-MS. This means that based on the chemical nature (e.g. protein, fat, small molecules), adulterants are screened in different mass regions. This will help to define if both technologies are complementary and best to detect milk adulterants or if one profiling methodology complemented with few targeted methods is a better solution.

\section{A Quick and Simple Quantitative Multi-analytes Method for the Detection of Nitrogenous-rich Compounds by LC-MS/MS}

Over the last years several fraud cases involving high-nitrogen content chemicals were observed raising safety and compliance issues in the Food Industry. Such compounds were added into food raw materials (e.g. milk, wheat gluten) increasing artificially their protein content for economically-motivated adulteration. Indeed, the official Kjeldahl method ${ }^{[26]}$ commonly used for protein analysis does not discriminate between endogenous nitrogen from protein sources and exogenous nitrogen fraudulently added. The 2008 Chinese milk scandal is a typical example of fraud- 


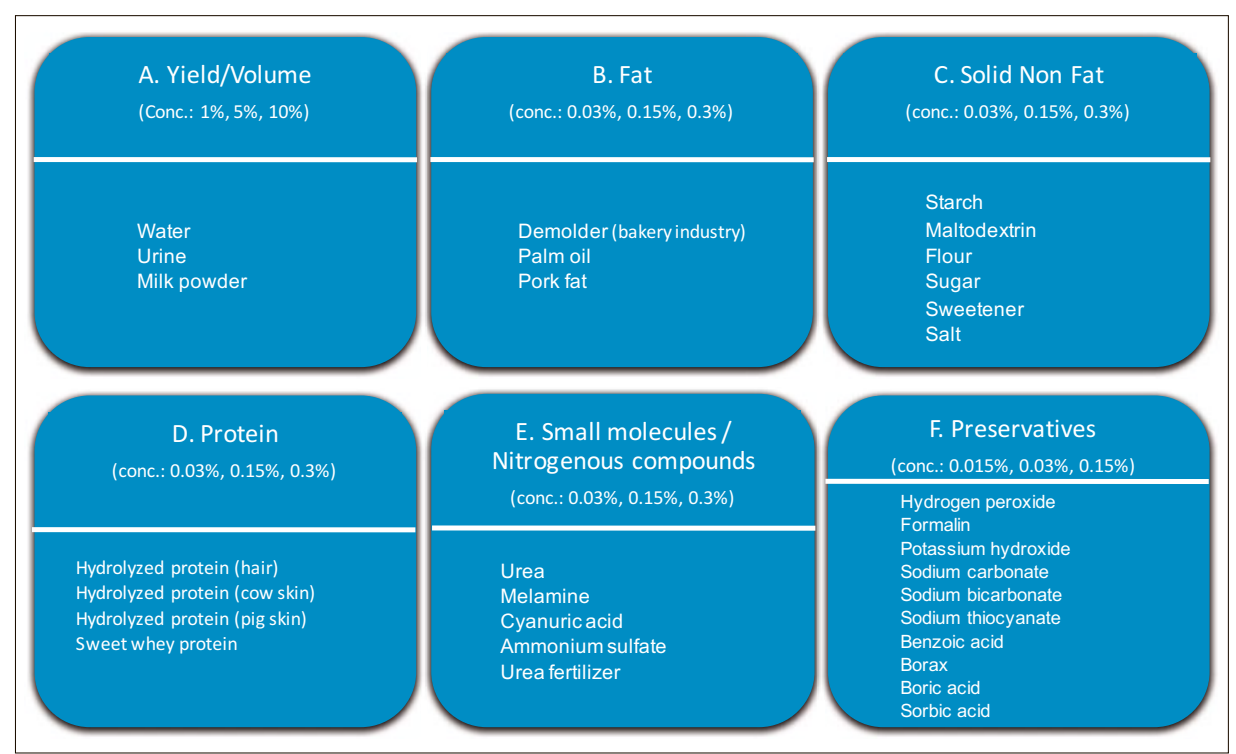

Fig. 5. Selection of milk adulterants for the FT-Mid-IR fingerprinting model validation. Based on the milk payment criteria (volume, fat, protein, solid non fat and microbiological quality), six categories of adulterants were created and 31 compounds tested at concentrations compatible with economic gain.

ulent use of melamine in milk causing the illness and death of young infants.

Although several analytical methods for nitrogenous compound detection have been already published, none of them are applicable as such for quality control operations. Either such methods cover a large number of compounds but provide qualitative results with high limits of detection in a limited number of matrices ${ }^{[27]}$ or they describe quantitative methods able to reach lower limits of quantification, but cover only a limited range of compounds. ${ }^{[28,29]}$ In addition, the sample preparations proposed are often time consuming and therefore not fit for high-throughput routine operations.

Consequently, a fast and simple multicompound method was developed, capable of detecting either high levels of nitrogen-rich compounds resulting from adulteration or low levels due to accidental contamination. This procedure covers fourteen nitrogenous compounds including melamine, ammeline, ammelide and cyanuric acid, nicknamed the 'big four'. This method encompasses a simple and fast sample preparation based on dilution and clean-up by dispersive solid-phase extraction. After separation with hydrophilic interaction liquid chromatography, detection of analytes is carried out in one single run by liquid-chromatography tandem mass-spectrometry (LC-MS/MS) using positive/negative switching in multiple reaction monitoring mode. Quantification is performed by the isotopic dilution approach using labelled internal standards. Validation was performed according to the European SANCO 12571/2013 ${ }^{[30]}$ requirements on various food matrices such as powdered infant formulas and infant cereals, liquid milk, dairy ingredients, raw cereals and meat and fish powders. Limits of quantification were typically between $0.05 \mathrm{mg} / \mathrm{kg}$ and $0.20 \mathrm{mg} / \mathrm{kg}$ depending on the analyte. Applicability of this method was further demonstrated by its successful implementation in a service laboratory.

\section{Conclusions}

As shown in this article, it is important to better understand the vulnerabilities in the food chain towards adulteration. Looking at the raw materials or ingredients inherent vulnerabilities, the possibilities to adulterate are infinite in terms of compounds which may be added intentionally to food. However, the type of fraudulent practices, e.g. unapproved enhancement of specific ingredients, raw material concealment and others, are much more limited. Therefore, it is worth while taking the time to perform first an in-depth assessment of the major factors influencing raw materials inherent vulnerabilities. This will help to evaluate the gaps and needs for new development of targeted or untargeted analytical methodologies for the deterrence and detection of raw materials adulteration. However, analytical surveillance is complex and can only be used to verify that the food authenticity process including raw material transparency and traceability is efficient. In this context, a good knowledge of the supply chain in terms of length, complexity, accessibility and with regard to specific raw materials traceability and supplier confidence is a key pre-requisite to mitigate the risk of food fraud.

Received: December 22, 2015
[1] B. Wilson, 'Swindled - the dark history of food fraud, from poisoned candy to counterfeit coffee', Princeton University Press, Princeton, NJ, 2008.

[2] GMA, Grocery Manufacturers Association and A.T. Kearney, http://www.gmaonline. org/downloads/research-and-reports/ consumerproductfraud.pdf, accessed August $15,2012$.

[3] J. Spink, D. C. Moyer, J. Food Sci. 2011, 76, 9.

[4] J. X. Liu, L. L. Wang, J. Liu, J. P. Wang, Food and Agri. Immun. 2014, $25,2$.

[5] T. T. Ng, P. K. So, B. Zheng, Z.P. Yao, An. Chem. Acta 2015, 884, 15.

[6] J. Spink, D. C. Moyer, Food Technol. 2013, 67, 1

[7] K. Everstine, J. Spink, S. Kennedy, J. Food Protection 2013, 76, 4 .

[8] C. Donaldson, Perspectives in Public Health 2014, 134,6

[9] J. C. Moore, J. Spink, M. Lipp, J. Food Sci. 2012, 77. R108.

[10] J. Spink, C. T Elliot, K. Swoffer, Food Sci. Technol. 2013, $27,4$.

[11] T. M. P. Cattaneo, S. E. Holroyd, J. Near Infrared 2013, 21, 5.

[12] M. Stoyke, F. Hamann, W. Radeck, P. Gowick, J. of Verbraucherschutz und Lebensmittelsicherheit 2013, $8,1$.

[13] M. A. Sentandreu, E. Sentandreu. Food Res. Int. 2014, 60, 19 .

[14] H. Hird, J. Chisholm, A. Sanchez, M. Hernandez, R. Goodier, K. Schneede, C. Boltz, B. Popping, Food Addit. Contam. 2006, 23, 645.

[15] M. E. Ali, M. A. Razzak, A. B. Abd Hamid, Food Anal. Methods 2014, 7, 1933.

[16] Reuters, http://www.reuters.com/article/2014/ 01/02/us-walmart-china-idUSBREA0103 O20140102, accessed January 5, 2014

[17] A. P. Bejing, http://news.yahoo.com/rat-meatsold-lamb-latest-china-food-scandal182459173.html, accessed May 4, 2013.

[18] A. N. Iwobi, I. Huber, G. Hauner, A. Miller, U Busch, Food Anal. Methods 2011, 4, 389.

[19] D. Steinke, R. Hanner, Mitochondrial DNA 2011, 22, 10 .

[20] N. V. Ivanova, T. S. Zemlack, R. H. Hanner, P. D. N. Hebert, Mol. Ecol. Notes 2007, 7, 544.

[21] R. H. Hanner, S. Becker, N. V. Ivanova, D. Steinke, Mitochondrial DNA 2011, 22, 106.

[22] Food and Drug Administration, http://www.fda. gov/downloads/Food/FoodScienceResearch/ UCM238026.pdf, accessed January 5, 2014

[23] A. O. Tillmar, B. Dell'Amico, J. Welander, G. Holmund, PLoS One 2013, DOI: 10.1371/ journal.pone. 0083761

[24] A. Afzal, M.S. Mahmood, I. Hussain, M Akhtar, Pakistan J. Nutrition 2011, 10, 12

[25] D. I. Ellis, V. L. Brewster, W. B. Dunn, J. W. Allwood, A. P. Golovanov, R. Goodacre, Chem Soc. Rev. 2012, 41.

[26] AOAC Official Method 955.04, 1995.

[27] G. Abernethy G, K. Higgs, J. Chrom. A 2013, $1288,10$.

[28] S. MacMahon, T. H. Begley, G. W. Diachenko, S. A. Stromgren, J. Chromatog. A 2012, 1220 , 101.

[29] J. Draher, V. Pound, T.M. Reddy, J. Chromatog A 2015, 1373, 106.

[30] SANCO/12571/2013, 'Guidance document on analytical quality control and validation procedures for pesticide residues analysis in food and feed', http://www.eurl-pesticides.eu/ library/docs/allcrl/AqqGuidance Sanco 2013 12571.pdf 